\title{
Hacia el límite ético de la estrategia: poniendo la ética en el centro de las decisiones en la empresa
}

Towards the ethical limit of strategy: putting ethics at the center of company decisions

Rumo ao limite ético da estratégia: colocar a ética no centro das decisões da empresa

\author{
Jose Daniel BARQUERO CABRERO \\ ESERP Business \& Law School, España / jd.barquero@eserp.com \\ Francisco Javier GARRIDO MORALES \\ The Royal European Academy of Doctors, RAED \\ francisco.garrido.oba@said.oxford.edu \\ Mercedes CANCELO SANMARTIN \\ Universidad de Málaga / cancelo@uma.es
}

Chasqui. Revista Latinoamericana de Comunicación

N.o 146, abril-julio 2021 (Sección Diálogo de saberes, pp. 303-322)

ISSN 1390-1079 / e-ISSN 1390-924X

Ecuador: CIESPAL

Recibido: 06-05-2019/Aprobado: 13-04-2021 


\title{
Resumen
}

La ética ocupa un papel central tanto en la en las enseñanzas y expresiones tradicionales de las Escuelas de Pensamiento Estratégico milenarias, como en el ámbito empresarial aplicado a la realidad del Siglo XXI. Desde los fundamentos antropológicos (Melé y Cantón, 2015) se encontraría tanto arraigada en los "fundamentos antropológicos de la dirección de empresas"[1], como en las expresiones de anticipación, colaboración y creatividad (Garrido y Mintzberg, 2019) que "fundamentan la persistencia de los temas estratégicos través de la historia relatada"[2].

Sobre esto Cortina (2013) nos recuerda que las personas "pueden ser más o menos morales según determinados códigos, pero todas tienen alguna estatura moral"[3] lo que viene muy a cuenta cuando consideramos dos grandes Escuelas de Pensamiento Estratégico como lo son la Oriental y la Occidental, como cuando exploramos la esencia milenaria de la enseñanza estratégica y encontramos una coincidencia fundamental con la ética en "la búsqueda de la excelencia" (Melé, 2012). En ellas descansan las fuentes para aproximarnos a una pregunta tan importante como inadvertida: ¿es la ética el límite último de la estrategia?.

Palabras clave: ética, empresa, comunicación

\begin{abstract}
Ethics occupies a central role both in the traditional teachings and expressions of the Millennial Schools of Strategic Thought, as well as in the business environment applied to the reality of the 21st century. From the anthropological foundations (Melé and Cantón, 2015) it would be found both rooted in the "anthropological foundations of business management" [1], and in the expressions of anticipation, collaboration and creativity (Garrido and Mintzberg, 2019) that "base the persistence of strategic themes throughout the story told" [2].

Regarding this, Cortina (2013) reminds us that people "can be more or less moral according to certain codes, but they all have some moral stature" [3], which is very important when we consider two great Schools of Strategic Thought such as the Eastern and Western, as when we explore the millenary essence of strategic teaching and find a fundamental coincidence with ethics in "the search for excellence" (Melé, 2012). In them lie the sources to approach a question as important as it is inadvertent: is ethics the ultimate limit of strategy?.
\end{abstract}

Keywords: ethics, business, communication

\section{Resumo}

A ética ocupa um lugar central tanto nos ensinamentos e expressões tradicionais das Escolas Milenares de Pensamento Estratégico, como no ambiente de negócios aplicado à realidade do século XXI. A partir dos fundamentos antropológicos (Melé e Cantón, 2015) encontraria-se tanto enraizado nos "fundamentos antropológicos da gestão empresarial" [1], e nas expressões 
de antecipação, colaboração e criatividade (Garrido e Mintzberg, 2019) que "embasam a persistência de temas estratégicos ao longo da história contada"[2]. A esse respeito, Cortina (2013) lembra que as pessoas "podem ser mais ou menos morais segundo certos códigos, mas todas têm alguma estatura moral” [3], o que é muito importante quando consideramos duas grandes Escolas de Pensamento Estratégico como o oriental e o ocidental, como quando exploramos a essência milenar do ensino estratégico e encontramos uma coincidência fundamental com a ética na "busca da excelência" (Melé, 2012). Neles estão as fontes para abordar uma questão tão importante quanto inadvertida: a ética é o limite último da estratégia?

Palavras-chave: ética, negócios, comunicação

\section{Introducción}

La ética ocupa un papel central tanto en la en las enseñanzas y expresiones tradicionales de las Escuelas de Pensamiento Estratégico milenarias, como en el ámbito empresarial aplicado a la realidad del Siglo XXI. Desde los fundamentos antropológicos (Melé y Cantón, 2015) se encontraría tanto arraigada en los "fundamentos antropológicos de la dirección de empresas"l, como en las expresiones de anticipación, colaboración y creatividad (Garrido y Mintzberg, 2019) que "fundamentan la persistencia de los temas estratégicos través de la historia relatada"2.

Sobre esto Cortina (2013) nos recuerda que las personas "pueden ser más o menos morales según determinados códigos, pero todas tienen alguna estatura moral" 3 lo que viene muy a cuenta cuando consideramos dos grandes Escuelas de Pensamiento Estratégico como lo son la Oriental y la Occidental, como cuando exploramos la esencia milenaria de la enseñanza estratégica y encontramos una coincidencia fundamental con la ética en "la búsqueda de la excelencia" (Melé, 2012). En ellas descansan las fuentes para aproximarnos a una pregunta tan importante como inadvertida: ¿es la ética el límite último de la estrategia?

Si consideramos la presencia declarada y documentada de estrategas en India y China hace ya aproximadamente 2500 años ${ }^{4}$, podríamos suponer una cierta coincidencia histórica en Oriente respecto de pensadores estratégicos tales como T’ai Kung, Sun Tzú, Sun Bin, Musashi, Miyamoto esto porque tanto la convergencia como la persistencia en las enseñanzas de las culturas milenarias

1 Melé, Domènec y González Cantón, César (2015), Fundamentos antropo- lógicos de la dirección de empresas, EUNSA, Pamplona.

2 Garrido, Francisco J.y Mintzberg, Henry (2019): "Pensamiento Estratégico: hacia el ADN de la Planificación Estratégica”. Oxford Publishing, Oxfordshire, UK.

3 Cortina, Adela (2013): "Ética". Paidos, Barcelona, España (2ed).

4 Garrido, Francisco J. (2015): "Determinantes de las Escuelas de Pensamiento Estratégico de Oriente y Occidente, y su contribución para el Management del Siglo XXI". Edit. Real Academia Europea de Doctores (RAED), Barcelona, España. 
y centenarias expresan raíces, puntos de apoyo y fundamentos que se expresan en nuestra raíces antropológicas comunes.

\section{Antecedentes Históricos de carácter ético y moral en la estrategia Oriental}

En la tradición de la Escuela de Pensamiento Estratégico orientales se estudian las enseñanzas de Tai Gong o T'ai Kung (Siglo XI a.C.) quien fue el primer duque gobernante del poderoso estado de Qi durante el período de la Primavera y Otoño del período de los "Estados Combatientes" en la antigua China y claramente el originador de sus tradiciones estratégicas. El rol de Tai Gong en el campo intelectual de la milicia y la estrategia de la antigua China fue inmenso. Si bien ante la mirada occidentales los tratados de Tai Gong sobre la estrategia o "Seis Enseñanzas Estratégicas Secretas" suelen ser citados solo como "uno de los Siete Clásicos Militares de la antigua China”, debemos tener presente que Tai Gong fue en efecto el padre del pensamiento estratégico militar chino.

De acuerdo con The Record of the Grand Historian (Shi Ji / Shih Chi), Tai Gong fue el jefe de la formulación e implementación de Zhou's (1046-256 d.C, estado el que Tai Gong servía) y de la estrategia global para destronar a la dinastía Shang (1600-1045 d.C.). Las estrategias y estratagemas chinas fueron modeladas en ese momento y por esto entre los historiadores orientales se dice que las estrategias militares chinas se originaron con Tai Gong. La visión ética y moral que el autor dibuja en las "Seis Enseñanzas Estratégicas Secretas" se expresan en el párrafo segundo (De la plenitud y el vacío) donde indica que la diferencia entre países caóticos y ordenados se debe "al gobernante, no a la suerte o a los dioses", por lo que este líder y estratega debe cimentar su grandeza a través "de la integridad y de cómo tratas a las personas", "recompensando a los que son leales y que respetan a los demás”, tendiendo a recompensar los buenos actos y prohibiendo el mal ${ }^{5}$. Es posible afirmar que estos influjos valóricos

Por su parte las enseñanzas del Maestro Sun o Sun Tzú tienen la marca a fuego de uno de los nombres más reiterados en la literatura estratégica, cuya obra arriba a Occidente una década antes del estallido de la Revolución Francesa, de la mano del misionero jesuita Jean Joseph Marie Amiot (1718-1793), quien además fue autor del primer diccionario Manchú-Francés (1789). Ha sido gracias al esfuerzo de traducción del padre Amiot (originalmente publicado como Los Trece Artículos y luego conocido como El Arte de la Guerra) fue reeditada en 1782 en Francia y Alemania, y luego Japón, Rusia, Inglaterra y España, ajustándose cada vez más al original ${ }^{6}$. No sin algunas controversias los historiadores orientales sitúan a Sun Tzú como nacido en el estado de Qi (China temprana del

5 Sawyer, Ralph D. (1997): "The Six Secret Teachings on the Way of Strategy". Shambhala Books, Bo. Colorado, USA.

6 La traducción del Padre Amiot s.J. no es considerada la más ajustada a los escritos originales chinos, lo que no resta en ella el gran valor que supone el esfuerzo por tender puentes entre las cosmovisiones orientales y occidentales. 
siglo IV a.C.), situando su vida en una época de gran influjo filosófico, técnico y artístico, así como una época de grandes batallas y guerras que terminarían con los siete reinos originales ( $\mathrm{Qi}, \mathrm{Chu}, \mathrm{Yao}, \mathrm{Han}$, Zhao, Wei y Qin). Entre los estrategas de Oriente es a Sun Tzú, a quien se atribuye la autoría del reconocido tratado sobre El Arte de la Guerra, donde el autor comienza por indicarnos que "la estrategia de trata de vida y muerte", así como de "supervivencia y destrucción": opuestos que se sitúan en el contexto de las enseñanzas orientales y que obligan a pensar en estas artes no solo como fundamentales para las relaciones humanas, sino como verdades trascendentes de la cosmovisión oriental situadas en las tradiciones de los opuestos. Así para el Maestro Sun la "victoria perfecta es la que no recurre a las armas", y el "mejor estratega es el que consigue lo que desea sin necesidad de combatir" y apunta sobre la importancia del trato "benevolente con el adversario", a fin de granjearse una cierta legitimidad y dejando entrever una cierta enseñanza ética aplicada. Melé (2013) nos recuerda que "para algunos profesores de dirección estratégica, el Arte de la Guerra de Sun Tzu es libro de cabecera, asimilando a los competidores al enemigo a batir. Otros no llegan a tanto, pero ven la estrategia como lo esencial para captar valor económico dónde lo haya", lo que describe claramente el influjo que este trabajo milenario ha tenido en sus traducciones para el mundo directivo.

Un aspecto muestra el valor que Sun Tzú asigna a las condiciones éticas y virtuosas en el ejercicio de la estrategia, se develan en el primer párrafo de su tratado:
Por lo tanto,
existen cinco factores
que debemos comparar
para poder evaluar
la situación.
El primero es el dao (道 Dáo)
El segundo es el cielo (田 Tian)
El tercero es la tierra (迪 Di)
El cuarto es el mundo (江 Jiang)
El quinto es el método (方法 $F a$ )

En este cuerpo de texto (segundo párrafo de El Arte de la Guerra) se pone de manifiesto la importancia del Tao o Dao, uno de los conceptos e ideogramas más populares de la cultura oriental en general y de la cultura china en particular. Ha sido traducido como "camino" o "vía", hay coincidencia entre los historiadores

7 Melé, Domenec (2013): ¿Dirección estratégica o dirección general? Una cuestión ética posted on noviembre 20, 2013 / Ética Empresairal, blog network. http://blog.iese.edu/eticaempresarial/2013/1²0/direccion-estrategica-vs-direccion-general-relevancia-etica/ (accessed March 22, 2019). 
más prestigiosos en terminologías que le vinculan con nuestro concepto de "verdad", "rectitud", "virtud" y "Ley moral"8 o "influencia moral"9.

Más tarde y en el seno de una familia de guerreros Wei nace quien ha sido conocido como Sun Bin (nacimiento no datado - 316 a.C.) autor de "El Arte de la Guerra II" Sun Tzú (a quien algunos historiadores signan como el "Sun mutilado" o el sobrino de Sun Tzú). Su trabajo es en cierto modo continuador del Arte de la Guerra, aun cuando Sun Bin había cursado estudios con Wang Li (conocido estratega taoísta de la época y autor del tratado estratégico El Maestro del Valle del Demonio) de quien habría adoptado sus preceptos morales: "las personas de baja catadura moral utilizarán la imitación de los demás de una forma perversa y siniestra, hasta el punto en que pueden destruir familias y usurpar países"10.

Debemos considerar que nos situamos en el período en que tradición china de gobierno comenzó a mostrar gran énfasis en el legalismo ${ }^{11}$ (tradición materialista y pragmática opuesta al Confucianismo, pero que no se aparta del Tao), a través de las tradiciones del maestro Shang Yang (390-388 a.C.), quien es responsable de la teoría del Ding Fa (定法) que posibilitó el ajuste de los estándares éticos en el ejercicio del poder y del Yi Min (一民), la que sistematizó e impulsó el trato a las personas en forma consistente. Estas políticas fueron reforzadas desde una perspectiva ética-pragmática centrada en los monarcas y aplicada a la enseñanza de los directivos públicos de la época por Han Fei (280233 a.C.).

A continuación encontramos los preceptos del Artha (o estado de riqueza material) de Chanaka Kautilya (estratega indio, contemporáneo de Aristóteles) en su trabajo Arthasastra (321-300 a.C.) donde el autor propone un equilibrio entre "rectitud $y$ riqueza" 12 como bases para la preservación de la buena administración de los recursos públicos, abundancia y riquezas. El texto presenta explícitas orientaciones de cómo "la ética colectiva puede mantener y preservar a la sociedad unida" y considerando "los valores éticos como el pegamento que une a la sociedad y promueve el desarrollo económico". Kautillya en el Arthashastra "mezcla el áspero pragmatismo por el que es famoso, con la compasión por los pobres, por las esclavas y por las mujeres. Revela la imaginación de un romántico al imaginar todo tipo de escenarios que difícilmente pueden darse comúnmente en la vida real"13. Consideremos que en general la tradición india del pensamiento estratégico enfatizaba el concepto de dharma como "un conjunto de reglas que unían al gobernante y a los gobernados por igual. Además, los conceptos indios de oficio tenían fuertes matices morales

8 Cleary, Thomas (2006): "El Alma del Samurai". Kairos, Barcelona, España.

9 Griffith, Samuel (1963): "The Art of War". Oxford University Press, Oxford, UK.

10 Cleary, Thomas (1993): "Thunder in the Sky: on the aqcuisition and exersise of power". Shambala, Boston, USA.

11 Roy, Kaushik (2012): "Hinduism and the Ethics of Warfare in South Asia: from antiquity to present". Cambridge University Press, NY, USA.

12 Shamasastry, R. (1915): "Kautilya. Arthashastra". Bangalore: Government Press, 515-520.

13 Paul Brians et al, eds. (1993). Reading About the World, Vol. 1. Washington State University Press. ISBN 0-15567425-0. Retrieved 2016-02-20. 
y éticos en contraste con el énfasis realista contemporáneo sobre el poder agregado y los factores materiales" ${ }^{\prime 14}$.

El aporte de la Escuela Japonesa de Pensamiento Estratégico es expresado por Miyamoto Musashi (1584-1645), quien en la primera mitad del siglo XVII escribe el Libro de los Cinco Anillos aplicando con la genialidad del autodidacta las enseñanzas para encontrar "la verdadera esencia del arte del combate" en el Bushido (texto del "camino del guerrero" que contiene preceptos de formación práctica y moral para el guerrero samurái japonés), para quien la deshonra y la falta a la moral es razón suficiente para aceptar la muerte. Mismas enseñanzas a las que aportaría más tarde Yamamoto Tsunetomo (1659-1719) dictando oralmente las enseñanzas de Hagakure a su discípulo Tashiro Tsuramoto a comienzos del Siglo XVIII, para quien "los buenos prosperan y los malos decaen tan solo como lección moralizante". Ambos japoneses consideraron el camino del estratega como un "aprender el camino de la muerte (Miyamoto) o como un aceptar la muerte" (Yamamoto)"15. En este sentido ambos refieren al concepto de honra a la hora de aceptar las decisiones propias y del destino.

Daidoji Yuzan (Siglo XVI) complementa estas centenarias enseñanzas escribiendo el Código del Samurai escribe un texto metódico para la formación de los jóvenes caballeros samuráis -quienes, como hemos indicado, se guiaban por los códigos éticos del Bushido- pero proponiendo un punto de conexión central con las enseñanzas de Sun Tzú: "el guerreo debe tener presente constantemente, por encima de todo, de día y de noche, que ha de morir", y agrega "si lo tiene siempre presente vivirá siguiendo los caminos de la integridad, el valor y el honor"16. El autor pone énfasis en la condición de una vida efímera a través de la que el guerreo samurái puede caminar fortalecido, evitando males y adversidades, al ser consciente de su impermanencia a través de la vívida presencia de la potencial muerte de su organización, decisiones y proyectos, a través de sus propias decisiones o a través de una derrota infligida por quienes nos enfrentan en el conflicto, o por nuestros competidores.

En la cosmovisión china e india temprana (siglo IV a.C.) o japonesa posterior (siglo XVI) se evidencian expresiones en las que la ética en la toma de decisiones estratégicas se acercan a las ideas del Tao (o "Libro del Camino"). Estas decisiones de los estrategas fluyen como parte de la energía amplia y unificada de funcionamiento universal, que no es completamente neutral: los caminos que provocan deben favorecer mutuamente a las personas y no causar daño. Los caminos escogidos pos los estrategas "serán expresiones un poder benéfico, una estructura o ley universal de tipo dialéctica, que se manifiesta en la dualidad del acontecer del mundo y con esa impronta marca la formación y el ejercicio de

14 Dr Arvind Gupta, Director General IDSA, and Professor Sven G. Holtsmark, Director, IFS, Oslo, 'Foreword', in Kanti Bajpai, Saira Basit and V. Krishnappa (eds), India's Grand Strategy: History, Theory, Cases, New Delhi: Routledge, 2014, p.x.

15 Oshima, Hitoshi (2011): "La Vía del Samurai”. La Sfera de los Libros. Madrid, España.

16 Schimcke, Don (2010): "El Ejecutivo Samurai”. Edaf, Madrid, España. 


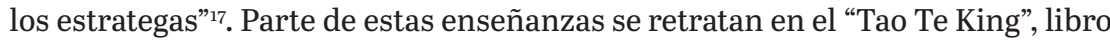
central del pensamiento taoísta que puede traducirse como "El libro del Camino y de su Virtud" (Lao-Tsé, 600 a.C. aprox.), donde se describe la fuerza del Tao -fuerza de contradicción y de orden del universo- y se aconseja sobre el modo de obrar que representa al "hombre santo" o sabio, ya sea en la vida diaria como en el ejercicio del gobierno o en la empresa. Para Lao-Tsé "el que domina a otros es fuerte; el que se domina a si mismo es poderoso" y un elemento que equilibra ese dominio se explica a través de decisiones que hoy llamaríamos éticas: "el Tao es universal; puede utilizarse para la derecha o para la izquierda" ${ }^{\text {". }}$. Esto significa que las leyes del universo pueden utilizarse en forma moral, inmoral o amoral, lo que depende del carácter y la capacidad de decidir en forma correcta (ética), y oportuna, de quienes toman decisiones.

Por su parte para el estudioso y estratega chino Wei Liao Tzú (370-319 a.C.) propone que los estrategas se deben involucrar en la guerra "solo cuando se justifica como una forma de restablecer la justicia del Tao", teniendo siempre en cuenta los principios del bien común. Su tratado El Arte de Dirigir fue acabado por sus discípulos entre el 206 y 201 a.C. En este tratado el autor propone que "el estratega debe estar preparado para no dejarse llevar por la cólera y debe ser puro para que no pueda ser corrompido por las riquezas". Para este autor el carácter moral, emocional y mental del estratega y sus tropas resulta más relevante que las condiciones del terreno y la estrategia, los que en sus ideas resultan secundarios.

Ya en pleno Siglo XX será Yukio Mishima (1925-1970) quien a través de la lectura del Hagakure (1968) traducido como "Oculto por las hojarasca" suponía la encarnación de los códigos tradicionales de las virtudes de los guerreros japoneses o "la ética del samurái en el Japón moderno" y en la vida contemporánea. En el texto base de su tratado la "ética y su aspecto estaban determinados por la preocupación de no parecer vergonzoso ni ruin antes los ojos del enemigo (...) por eso no se trata de una consciencia moral interiorizada e individual, sino de una moral que depende del exterior"19. En tal sentido Mishima refuerza la idea de que la moral del samurái le obliga a hacer un esfuerzo sobrehumano para nunca mostrarse cansado (a pesar de estarlo), siempre parecer sano (más importante que estarlo) y en todo momento mostrarse valiente.

17 Garrido, Francisco J. (2010): "El Alma del Estratega”. McGraw Hill, Madrid, España.

18 Tao Te King, versión de John C. H. Wood. Edaf, Madrid, 1993.

19 Sese, M y Rubio, C. (2013): "Yukio Mishima: la ética del samurái en el Japón moderno". Alianza Literaria. Madrid, España. 


\section{Antecedentes Históricos de carácter ético y moral en la estrategia Occidental}

En la Grecia temprana se le llamó "sabiduría del general" (strategike sophia o strategon sophia) al del destello de claridad que iluminaba asertivamente la sabia visión para la toma de decisiones que se esperaba del strategos o general de ejército. Así mismo Delfos -en la cuna de Occidente- apunta a la trascendencia de las obras que surgen desde el ser con su "conócete a ti mismo", lo que nos obliga a contemplar las condiciones elementales para la formación de un estratega que se precie y merezca dirigir personas en el campo de batalla, o en el escenario empresarial. Consideremos que si bien la guerra era una realidad siempre presente en la vida griega, como indica Momigliano (1954) fue a la vez "un foco de emociones, valores éticos, reglas sociales"

Uno de los más influyentes historiadores latinos por el talante moral de sus relatos fue Cayo Julio César es considerado como uno de los más grandes generales y estrategos de la historia (12 de julio del año 100 en el seno de una de las familias más distinguidas de Roma). Sus grandes dotes de historiador y estratega se expresan en su tratado Bellum Gallicum, donde explica que mantener las adecuadas "cohortes formadas según la disciplina y moral militar" ${ }^{21}$ era la mejor defensa en tiempos de guerra, con base en el apropiado liderazgo y en la excelencia de los procesos.

Por su parte Alejandro III de Macedonia o Alejandro Magno (356 a 323 a.C.), considerado uno de los más grandes generales de la historia desarrolló durante sus doce años de campaña una brillante política de fusión con los persas, luego de la conquista de Persépolis. Alejandro -así como Escipión el estrategos- hacía gala de su altura moral en sus relaciones con los conquistados ejercitando las virtudes de la epieikeia (moderación) y la philantropía (generosidad), elementos fundantes del ejercicio del conquistador o eudoxia: la correcta doctrina para un liderazgo ético del conquistador, en el contexto de la conquista y la guerra.

Uno de los estrategas militares más citados ha sido Napoleón Bonaparte (1769-1821), quien dotado de gran talento, capacidad de trabajo y liderazgo, es considerado desequilibrante para la historia del siglo XIX. Para Bonaparte "la moral es a lo físico como tres es a uno" lo que supone en él una búsqueda idealizada del hombre libre de patrones, que permita el perfeccionamiento moral de toda la humanidad. Asimismo creerá en el progreso individual y colectivo "asentando sus valores en el respeto, democracia, justicia y solidaridad entre los seres humanos".

Entre los teóricos occidentales más influyentes en las academia de Ciencias Militares surge la figura del Archiduque Carlos de Austria (cuyos trabajos nunca fueron traducidos al inglés y de allí su poca difusión), del táctico y escritor

20 A. Momigliano,"Some Observations on Causes of War in Ancient Historiography", Acta Congressu Msadvigiani Proceedings of the Second International Clongress of Classical Sltudies,1954, Copenhagen,1958,pp.199-21 21 Goya y Babuena (1982): "Bellum Gallicum, con notas de Napoleón Bonaparte”. Iberia, Barcelona, España. 
francés Paul Gédéon de Maizeroy (1719-1780), el suizo Antoine-Henri Jomini, el prusiano Carl von Clausewitz y el francés Count Guivert.

Por su parte Karl von Clausewitz, militar nacido en el Reino de Prusia quien a partir de su excelencia en el campo de batalla ha sido considerado como uno de los más influyentes estudiosos de la conducta y “visión” de los estrategas occidentales de todos los tiempos. Con una vida dedicada al estudio de las artes, ciencias, educación, filosofía (gran conocedor de la obra de Kant) y ética, dejó un importante legado a la Ciencia Militar moderna, principalmente a través de su conocido tratado de ocho volúmenes: "De la Guerra"22, escrito junto a su mujer Marie, quien colaboró de manera fundamental en la obra "como ayudante de investigación y redacción" ${ }^{23}$, y quien publica finalmente la obra en 1832, luego que von Clausewitz falleciera producto del cólera contraído en batalla.

Desde otra vereda Niccolò Machiavelli (1469-1527) considerado entre los grandes teóricos políticos y artistas literarios la civilización florentina occidental, nos legó en su trabajo The Art of War (1521) el concepto de virtú como el ideario de la "audacia, valentía, resolución y decisión" ${ }^{24}$ que un buen líder (político o militar) debe exhibir, sustentado con una innegable dosis de disciplina y entrenamiento (virtú ordinata): características que justifican unas virtudes civiles y políticas de una pragmática exacerbada, y mas bien lejanas a las virtudes religiosas y morales. En línea con estas inspiraciones renacentistas que impulsaron la expresión de fundamentales cambios en las reflexiones éticafilosóficas de la época, el autor se decanta en su escrito El Príncipe (1531) por explicar como "en las acciones de todos los hombres, y máxime en las de los príncipes, cuando no hay tribunal al que reclamar, se juzga por los resultados. Haga, pues, el príncipe lo necesario para vencer y mantener el Estado, y los medios que utilice siempre serán considerados honrados, y serán alabados por todos" (El príncipe, XVIII). En este sentido la inmoralidad de los actos aparece justificada como resultado de la importancia de los fines escogidos más allá de los medios utilizados para conseguirlos. En sus escritos el autor insta tanto a no mantener las promesas, como a no decir la verdad: es la exaltación del pragmatismo por sobre los valores y la moral, una fórmula que justifica la disolución ética de la palabra empeñada por los "señores" en función la inobservancia justificada de los acuerdos. Sobre esto Melé nos recuerda que si bien "la estrategia es siempre medial, es estrategia 'para' lograr un objetivo, generalmente económico; y lo que importa es tener éxito en aquello que se ha fijado" ${ }^{25}$, esto no supone en caso alguno que se puedan superar los límites éticos que nos demanda la acción correcta.

Las implicancias para la tradición y formación estratégica occidental estaría según Aranguren en que "un maquiavelismo confesado se anularía en

22 Cláusewitz, Carl Von (1976): "On War". Princeton University Press, Princeton NY, USA

23 Eftimova Bellinger, Vanya (2017): "Marie von Clausewitz: The Woman behind the Making of On War". W\&C, Washington, USA.

24 Wood, Neal (2001): "The Art of War". Da Capo Press, Cambridge, MA, USA

25 Melé, Domenec (2013): Op. Cit. 
cuanto tal: para ser eficaz tiene que se hipócrita y rendir tributo a la virtud"26, sentencias y recomendaciones que junto a otras hicieron que el Cardenal Reginal Pole, Arzobispo de Canterbury (1539) sindicara a Machiavelli como “enemigo del género humano", idea secundada por el Papa Pablo IV Carafa (1559) quien incluyó las obras del autor en la lista de censurados por la iglesia católica (donde permaneció hasta 1966), razón por la que Inocencio Gentillet consigue mayor fama con su Discurso sobre los medios de gobernar bien: anti-Machiavelo texto publicado en 1576. En la línea opuesta se planteó el ilustrado Jean-Jacques Rousseau (1712-1778), para quien Machiavelli habría sido un autor honesto y buen ciudadano, víctima de una lectura a lo menos superficial y corrupta hasta la época, defendiendo la profundidad de sus ideas y ofreciendo una imagen del autor como la de "un intelectual capturado por la Casa de Médicis" ${ }^{27}$, idea que comparte David Hume (1711-1776) quien en sus Discursos Políticos (1752) define a los trabajos de Machiavelli como los "de un genio y de gran juicio", positiva visión que compartirá con Carl Marx, Benedetto Croce y Benito Mussolini.

\section{4. Ética y Estrategia en el Siglo XXI: entre la integridad y el arte del engaño}

Sobre las y los directivos del Siglo XXI Robert Greene (2012) expresa que son una camada de estrategas que expresan "un punto alto del potencial humano" y por ello quienes se forman para desempeñarse en tareas directivas no solo deben aspirar a ser "jugadores de alto desempeño", sino además modelos de conducta para sus colaboradores.

Es por esto que si considerásemos la importancia de una sana "musculatura ética" las organizaciones, como fundamento para un apropiado desarrollo de soluciones serias y a la altura que el ejercicio de la vida humana y organizacional (pública y privada) en todo el planeta, daríamos por bienvenidas las palabras de Downs (1975) para quien la integridad es un fenómeno esencial de las buenas relaciones, dado que el engaño -por el contrario- desfigura la coherencia esencial "entre las realizaciones y las declaraciones". Por cierto que los numerosos engaños y fraudes locales de impacto global en América ${ }^{28}$ y Europa $^{29}$ en los últimos años han llevado a muchos autores y pensadores a conceptuar a las compañías multinacionales como entidades "enfermas".

Ante este panorama, cabe preguntar ¿Cómo es posible que errores financieros graves (mal llamada creatividad contable) hayan perdurado durante años en empresas tan grandes y avaladas por importantes firmas de auditoria contable?, ¿es posible pedir la confianza ilimitada de los accionistas en una

26 Aranguren, José L. (1996): “Ética y Política”. Biblioteca Nueva, Madrid, España.

27 Boriaud, Jean-Ives (2016): “Nicolás Machiavelo”. El Ateneo, Buenos Aires, Argentina.

28 Cfr. Artículos publicados sobre Enron en Diciembre de 2001 por The Wall Street Journal y en Diciembre 2003 Financial Times,

29 Cfr. Artículos publicados sobre Parmalat en Enero 2004 II Sole 24 Ore (fraude que ascendió a 14 mil millones de euros), o el fraude de Volkswaguen Alemania expuesto en Enero de 2017. 
empresa después de hechos tan significativos? La situación de opacidad es crítica si a ello se une la situación inestable de los mercados bursátiles, que ha llevado a descubrir importantes fracturas éticas dentro de las organizaciones públicas y privadas. ¿Pero es posible que una sola persona -un bróker-haya originado unas perdidas de 4.900 millones de euros al banco francés Société Générale en enero de $2008^{30}$ ? Detrás de estos sucesos se descubren comportamientos impropios en cadena de responsabilidades que no corresponden sólo a los autores materiales sino al personal del entorno empresarial y los directivos donde éstos trabajaban. Sumado a esto también hemos asistido al descubrimiento de empresas cuyo comportamiento ético hacia los clientes ha sido engañoso ${ }^{31} \mathrm{y}$ ha supuesto un beneficio a largo plazo que no para de resonar en los oídos de los stakeholders ${ }^{32}$. En una sociedad atravesada por la complejidad global, los comportamientos individuales de las y los directivos incide con mayor fuerza e impacto en la situación local y mundial de las compañías.

En efecto, en palabras de Laporte y Marignani (2012) desde el punto de vista ético es posible distinguir diversos niveles de impacto de los comportamientos humanos en el ámbito empresarial, donde en el primer nivel encontramos aspectos estrictamente individuales del trabajo y de la realización personal ${ }^{33}$; lo que podemos entender como "la vertiente individual del trabajo de los empleados y directivos en el ejercicio de la actividad, que requiere unos principios de actuación sólidos" ${ }^{34}$. En el segundo nivel podríamos distinguir los comportamientos éticos relacionados con otras personas, grupos y divisiones organizacionales en el ejercicio de cada actividad, así como el reparto y el ejercicio del poder (donde juegan un rol la responsabilidad, las relaciones honestas, la gestión transparente, como las adecuadas y justas atribuciones de méritos, ente otras).

En este sentido se desprende que los comportamientos poco éticos se manifiestan en los directivos a través de las tiranías personales, la deshonesta relación con los empleados, el injusto balance entre las prioridades personales y las del bien común.

En el tercer nivel representa el impacto que la empresa causa en la sociedad. Considerando que las empresas son personas es evidente que las decisiones que toman las y los directivos influyen y conducen decisivamente el modo en que la empresas actúan e impactan a la sociedad ${ }^{35}$, dado que desde tiempos milenarios

30 Cfr. Los artículos publicados del 25 al 31 enero de 2008 sobre Jérôme Kerviel en The Economist, Financial Times, II Sole 24 Ore.

31 Case_Study_The_Volkswagen_Emission_Scandal, researchgate.net/publication/323998958.

32 Cfr. Philip Holden, Ética para managers, (Barcelona: Paidós 2000), pp. 144 y ss. Es historico el caso Tylenol y Jonson\& Jonson, Cfr. Paul A Argenti and Janis Forman The Power of Corporate Communication : Crafting the Voice and Image of Your Business (New York: McGraw-Hill 2002), p.35.

33 Sobre la realización personal, Cfr. ARISTÓTELES, Ética a Nicómaco, Libro I.

34 Laporte y Marignani (2012): “Ética y Empresa”. En Garrido, FJ: “Lo que se Aprende en los Mejores MBA del Mundo”. Gestión 2000, Barcelona, España.

35 Cfr. A. Kakabadse and M.Morsing (Editors), Corporate Social Responsibility. Reconciling Aspiration with Application, (Palgrave: Macmillan 2006). 
es una tarea ineludible e inexcusable que de los directivos y estrategas lleguen a las resoluciones que las complejas situaciones derivadas de sus cargos les imponen: "tanto en el mundo de los negocios, como en el campo militar hay una expresión que explica la razón por la que se busca a las y los estrategas para comandar las operaciones: toma de decisiones. Es obvio que la implementación de una estrategia requiere de alguien que asuma riesgos (controlados, pero riesgo al fin) y que finalmente tome decisiones" ${ }^{36}$.

En efecto, la toma de decisiones es una función central en el ejercicio de las y los estrategas: viene con el cargo. Y dado que la función central de tales decisiones es la de asegurar un cierto "modelo de futuro"37 para las organizaciones" e influir $\mathrm{y}$ afectar a la sociedad misma, el modo en que quienes han sido escogidas y escogidos para llevarlas a cabo, así como su necesaria y férrea formación ética, resultan fundamentales para la sociedad del Siglo XXI.

Ya hemos visto que, por ejemplo: la decisión de lanzar al mercado un producto financiero riesgoso y sin respaldo, y que necesita de soberbios controles de transparencia y liquidez, conlleva serias implicaciones éticas porque está en juego no sólo la solvencia de las empresas involucradas y del sistema financiero, sino la garantía de seguridad y los ahorros de cientos de miles de personas. El efecto entre el divorcio entre la ética directiva y la estrategia de las compañías es lo que vivimos en la llamada "crisis subprime" de $2008^{38}$.

Si bien es cierto que en una sociedad que se debate entre lo local y lo global a inicios del Siglo XXI que supone unos imperativos éticos asociados a una cierta uniformidad legal, coincidimos con Marigniani y Laporte (2012) en que "no parece acertado que como presupuesto de partida se identifique lo ético como lo legal" 39 . Es por esto que los autores aclaran que si bien en el ejercicio estratégico en la empresa se deben tomar decisiones éticamente adecuadas, pero no siempre la ética y la eficacia van de la mano, dado que el hecho de que una acción sea ética no garantiza en sí misma que pueda ser la acción más adecuada para resolver un problema. La toma de decisiones debe ejercitarse ante una variedad de opciones pero siempre descartando los comportamientos poco éticos, debiendo distinguir las mejores opciones para la construcción y diseño de la estrategia: su formulación. Para esto y como antecedente previo, se pueden y deben evaluar moralmente los objetivos que guían el diseño de dicha formulación estratégica. Siguiendo a Melé (2013) diremos que el "para qué" de la estrategia debe ser resuelto encarando los posibles dilemas éticos que se nos plantean a partir de una apropiada formulación y evitando los facilismos que hacen suponer a algunos que lo "incorrecto" o el "engaño" sean propio de una mal entendida naturaleza estratégica. A esto es a lo que Ciappei (2006) llama a

\footnotetext{
36 Ricart, Joan E. \& Garrido, Francisco J.. 2015. “Estrategia y Dirección Estratégica: en busca del eslabón perdido”. Asian Journal of Business and Management (ISSN: 2321 - 2802) Volume 03 - Issue 05, October. 37 Garrido, Francisco J. (2018): "Modeling the Future". Oxford Strategy Business Press, Oxford, UK. 38 Lewis, Kay, Kelso \& Larson. 2010. "Was the 2008 Financial Crisis Caused by a Lack of Corporate Ethics?". Global Journal of Business Research, Vol. 4, No. 2, pp. 77-84, 2010 39 Laporte y Marigniani (2012): Op. Cit.
} 
el realismo estratégico de la empresa del Siglo XXI, que es lo que en sus palabras "permite conciliar los valores y las exigencias empresariales sin menoscabo de la eficacia o del beneficio"40.

\section{La ética en los programas de dirección de negocios y MBA}

En función de los desafíos actuales en la formación de los directivos cabe relevar la pregunta de Mintzberg (2004, pp. 73) sobre "si los programas de MBA preparan y están en condiciones de formar líderes capaces de asumir las responsabilidades que las empresas tienen frente a la sociedad" 41 , o si como indican Barquero y Oesterman (2013, pp.111) si los MBA "proveen a los estudiantes con un amplio sentido de ellos mismos como profesionales y ejecutivos" ${ }^{22}$. Esto porque iniciamos desde el supuesto que cuando formamos a una o un directivo en un programa de MBA les estamos formando como estrategas ${ }^{43}$, o tomadores de decisiones de impacto en el futuro de las organizaciones y sociedades en el mundo entero.

En nuestros propios análisis desde el Harvard Business School Corporate Level Strategy Group ${ }^{44}$ coincidimos en que los inmensos mercados y sociedades, con millares de millones de personas (como ocurre en África, China o India) que son impactadas por nuestras multinacionales, se multiplican y crecen no solo unas gigantescas oportunidades sociales y comerciales, sino a la vez unos inmensos desafíos éticos sobre las y los directivos de nuestras empresas, así como sobre los desempeños de sus decisiones, que desde a altura de la alta dirección no pueden ser otros que estratégicos y de alto impacto en el futuro de las naciones en las que se opera.

Ya hemos indicado en trabajos anteriores que sabemos que "los programas de administración y gestión de empresas son conscientes del desafío ético que afrontan los directivos y líderes de las organizaciones" 45 y haciendo eco de las demandas sociales y de los stakeholders han buscado introducir -en mayor calidad y cantidad- los contenidos y materias específicas que las sociedades globales demandan. Coincidimos en que la finalidad de la enseñanza es la formación de personas completas o profesionales conscientes, podemos coincidir en lo muy nociva que resulta una instrucción que "crea el poder de la competencia sin profundizar en la dirección moral que guía y conduce su

40Cfr. Cristiano Ciappei (2006): "Il realismo strategico nel governo d'impresa. Materiali per una pragmatica del valore". Firenze University Press. Firenze, Italy (pp. 63-64).

41 Henry Mintzberg, Managers not MBAsManagers not MBAs (USA: Berrett Koehler 2004). Minztzberg señala un error de fondo en la educación actual, pues acceden a los MBAs personas jóvenes con poca experiencia profesional, que piensan ser directivos después de la educación recibida, no obstante -según el autor- no cuentan con la suficiente experiencia en el "mundo real".

42 Barquero, J.D. y Osterman, P. (2013): “Nuevas Fronteras del Management”. Furtwanguen, Barcelona, España.

43 Garrido, Francisco J. (2018): "Modeling the Future". Oxford BS Publishing, Londres, UK.

44Garrido es Miembro Fundador del Grupo de Estrategias de Nivel Corporativo de Harvard Business School (Boston, MA).

45 Garrido, Francisco J. (2012): Op. Cit. 
empleo" ${ }^{46}$. Al respecto, hemos visto en nuestros trabajos sobre la formación de los MBA del Siglo XXI como la disciplina ética en algunas de las mejores escuelas de negocios de Europa y Estados Unidos (conocidas por calidad y publicaciones especializadas ${ }^{47}$ ), donde si bien cada año cambian sus posiciones en los rankings, estudios y guías comparativas con diversas escuelas del planeta que dictan estos programas para directivos, suelen mantenerse las que Laporte (2012) identifica y ubica siempre "entre los primeros puestos". A partir del tipo de colaboración activa que los investigadores encontraron por parte los programas, describen cómo la disciplina de ética se perfila en los programas de MBA Harvard Business School (U.S.A.); University of Pennsylvania: Wharton (U.S.A.); Stanford University Graduate Business School (U.S.A); London Business School (UK); Northwestern University: Kellogg (U.S.A.); Yale School of Management (U.S.A.); Columbia Business School (U.S.A.); New York University: Stern (U.S.A.); MIT: Sloan (U.S.A.); Dartmouth College: Tuck (U.S.A.); IESE Business School (España); ESADE Business School (España); IE Business School (España); ESIC (España) ${ }^{48}$.

Si bien el análisis fue realizado de acuerdo con parámetros de referencia que han pasado por verificar la existencia o no de cursos dedicados a la ética, valores y comportamiento de dirección, responsabilidad social de la empresa o responsabilidad profesional, se debe considerar que el grupo investigado incluye programas MBA realizados a tiempo completo y generalmente de más de un año y medio de duración, comparado la terminología empleada para denominar los cursos, la posición que ocupa dentro del programa MBA, el syllabus del mismo, la formación del profesorado que dicta tales asignaturas, el tipo de metodología y la bibliografía.

Por nuestra parte y a través las variables y reportes que exploramos para elaborar nuestra "Guía Elegir un MBA 2019-20"49 hemos visto como entre las 120 escuelas que hemos estudiado en los cinco continentes (ver anexo), solo la mitad de ellas incluyen el término "ética" en el nombre de ciertos cursos específicos, hecho que refleja una cierta consistencia entre la muestra del estudio referido (2012) y nuestros datos actualizados. A modo de ejemplo, los nombres de los cursos se expresan del siguiente modo: Wharton (Ethics and Responsibility), Stanford (Ethics in Management), London Business School (Ethics and Corporate Responsibility), MIT (Ethical Practice: Professionalism, Social Responsibility and The Purpose of the Corporation), IESE (Leadership and Ethics, Business Ethics), EXEC (Ética y Empresa), Darmouth (Ethics in action) y ESIC (Ética Empresarial).

46 Cfr. J.S. Dickey, citado por Stephen R. Covey, L'ottava regola. Dall'efficacia all'eccellenza (Milano: Franco Angeli 2005).

47 Escuelas referidas como tales por Financial Times (The Global MBA rankings FT), The Economist (Which MBA), US News (The MBA Guide for America's) y Guía Elegir un MBA (Planeta DeAgostini)

48Se agradece las informaciones e ideas aportados por los siguientes profesores: a) de Harvard Business School: Joseph Badaracco, Joshua Margolis y Alison Cominos; b) de London BS, Diana Robertson; c) de Yale School of Management, Prof. Daylian Cain; d) de Columbia, Raymond Horton; e) de MIT Sloan, Prof. Leigh Hafrey; f) de IESE, Domènec Melé y Joan Fontrodona.

49 Garrido, Francisco J. (2019): “Guía Elegir un MBA”. Edit. Gestión 2000, Barcelona, España. 
Otras escuelas emplean denominaciones tales como Leadership, Social Responsibility o Global Values.

Si bien aun hay escuelas como Harvard Business School (Estados Unidos) que incluyen dos cursos obligatorios de ética en su MBA, la denominación de los mismos se presenta más bien con el término Leadership. En el caso de IESE Business School (España) ofrece un curso obligatorio en el second term denominado "Business Ethics", además de unos cursos adicionales en el segundo año sobre "ética y finanzas" e "implicancias éticas y sociales del cambio tecnológico”, lo que desde la persperctiva de Kellogg (Values-based Leadership), Yale University (Leadership and Values) y Desautels McGill University (Global Leadership).

Por otro lado al observar la posición que ocupan los cursos de ética en cada programa de MBA y desde la perspectiva de su opcionalidad u obligatoriedad, la tendencia general es la de ubicar los cursos de ética entre las enseñanzas obligatorias de la formación central o materias troncales, pero no hay una ubicación estándar al inicio o al final de los programas, que pueda ofrecer signos de preferencia o acuerdo entre las diferentes escuelas. Entre las 120 escuelas de negocios estudiadas, el $71 \%$ de ellas ofrecen a lo menos un curso obligatorio de Business Ethics, con una clara posición dentro del programa. Un $27 \%$ de ellas ofrecen más de un curso (también obligatorio) y un $12 \%$ ofrece cursos de ética especializada en determinadas industrias (bioética, sector público y otros).

En el caso de los docentes la titularidad de los cursos varía mucho de año en año y por esta razón los profesores se valoran con frecuencia más en función de sus intereses en el campo de la investigación relacionado con la ética que por la enseñanza efectiva del curso. En esto coinciden los trabajos de Walckot (2016), quien informa que los profesores del curso de ética entre las escuelas de negocios del top ten de The Economist y del US News, en general son los de mayor rotación entre los equipos académicos y tienden a vincularse mucho menos con departamentos de Business Ethics (en general vienen de General Management).

\section{Conclusión}

Para algunas y algunos directivos el único parámetro ético se relaciona con el marco regulatorio o legal, y es por esto que incluso suponen que el ejercicio de la Responsabilidad Social en sus organizaciones se resuelve con haber cumplido con los mínimos legales de las sociedades que les acogen. Estas mentalidades legalistas también inciden -de acuerdo a los resultados evidenciados por el estudio- en el desarrollo de algunas miopías estratégicas que destruyen valor en las relaciones con la comunidad.

Es nuestra opinión que los estrategas occidentales se verán beneficiados con una formación que les permita involucrarse personalmente en los temas morales y éticos, y que les permita ejercitar sus condiciones en la lucha por adecuar sus comportamientos a los valores en los que les hayamos formado. 
Siguiendo a Holden (2001) podemos ejemplificar numerosos casos en que las estrategias de buena ciudadanía empresarial y los beneficios económicos van de la mano en el mediano y largo plazo, sobre todo porque aseguran una mayor sustentabilidad de los proyectos en el tiempo.

La complejidad de la combinación entre ética y estrategia supone una complejidad que no es tal, pues descartada esta sola limitación para el diseño, formulación o ejercicio estratégico, tenemos todas las demás posibilidades disponibles y a la mano en el mundo de la creatividad y la innovación del siglo XXI (lo que no es poco). Es por esto que si desarrollamos apropiadamente las cualidades propias de los líderes y directivos éticos en nuestros estrategas, estaremos no solo influyendo en quienes diseñan el futuro de nuestro mundo a valor presente, sino aportando para que ese sea un mejor futuro.

Así las perspectivas modernas no se limitan a proponer a la ética como un conjunto de prohibiciones o como un manual para resolver dilemas en el ámbito de las corporaciones, sino como una "guía para la excelencia humana en las organizaciones empresariales"50 y un adecuado cauce "para la idea de perfeccionamiento" ${ }^{11}$ que es propia de los aportes originarios de la estrategia milenaria. En este trabajo hemos recorrido también las perspectivas antropológicas que explican las expresiones de la ética como actividades intrínsecas a toda acción humana y por lo tanto -podemos agregar- también propias de la humana búsqueda por modelar cada vez un mejor futuro.

50 Melé, Domenec (2009): "Business Ethics in Action: seeking human excellence in Organizations". Palgrave Mcmillan, London, UK.

51 Garrido, Francisco (2018): Op. Cit. 


\section{Anexo}

\begin{tabular}{|c|c|c|}
\hline $\mathrm{N}^{0}$ & Nombre Escuela & País \\
\hline & Amsterdam Business School & Holanda \\
\hline 2 & Ashridge Business Schoo & Reino Unido \\
\hline 3 & Aston Business School & Reino Unido \\
\hline 4 & Audencia Nantes Ecole de Management & Francia \\
\hline 5 & Babson F.W Olin Graduate School of Business & Estados Unidos \\
\hline 6 & Bath School of Management & Reino Unido \\
\hline 7 & Birmingham Business school & Reino Unido \\
\hline 8 & Boston University School of Management & Estados Unidos \\
\hline 9 & Bradford University Schoo of Management & Reino Unido \\
\hline 10 & Cambridge Judge Business School & Reino Unido \\
\hline 11 & Cass Business school & Reino Unido \\
\hline 12 & Chicago Booth School of Business & Estados Unidos \\
\hline 13 & china Europe International Business Schoo & China \\
\hline 14 & Chinese University of Hong Kong & China \\
\hline 15 & Columbia Business School & Estados Unidos \\
\hline 16 & crannield Schoo of Management & Reino Unido \\
\hline 17 & Curtin Graduate School of Business \& Law & Australia \\
\hline 18 & Duke Fuqua School of Business & Estados Unidos \\
\hline 19 & Darden Graduate school & Estados Unidos \\
\hline 20 & Desautels Faculty of Management & Canada \\
\hline 21 & Durham Business School & Reino Unido \\
\hline 22 & EAE Business School & España \\
\hline 23 & EDHEC Business Schoo & Francia \\
\hline 24 & EGADE Business Schoo & Mexico \\
\hline 25 & Eller college of management & Estados Unidos \\
\hline 26 & ESERP Business \& Law School & Espana \\
\hline 27 & ESADE Business Șchool & Espana \\
\hline 28 & Escola de Administracao de São Paulo, EAESP & Brasil \\
\hline 29 & EXEC Business School & España \\
\hline 30 & Fisher College of Business & Estados Unidos \\
\hline 31 & Foster School of Business & Estados Unidos \\
\hline 32 & Freeman School of Business & Estados Unidos \\
\hline 33 & Goizueta Business School & Estados Unidos \\
\hline 34 & Grenoble Graduate School of Business & Francia \\
\hline 35 & Haas School of Business & Estados Unidos \\
\hline 36 & Harvard Business School & Estados Unidos \\
\hline 37 & HEC School of Management & Francia \\
\hline 38 & Henley Business School & Reino Unido \\
\hline 39 & Henry $\mathrm{B}$ Tippie College of Business & Estados Unidos \\
\hline 40 & Hult International Büsiness School & Estados Unidos \\
\hline 41 & IAE Business School & Argentina \\
\hline 42 & IDE Business School & Ecuador \\
\hline 43 & IESE Busines School & Espana \\
\hline 44 & IIMAhmedabad & India \\
\hline 45 & IMD Business Schoo & Suiza \\
\hline 46 & INALDE Business School & Combia \\
\hline 47 & Imperial College Business School & Reino Unido \\
\hline 48 & INCAE Business School & Costa Rica \\
\hline 49 & INSEAD Business School & Francja \\
\hline 50 & IE Instituto de Empresa & Espana \\
\hline 51 & IPADE Business School & Mexico \\
\hline 52 & International University of Japan Graduate School & Japon \\
\hline 53 & ITAM & Mexico \\
\hline 54 & Johnson Graduate School of Management & Estados Unidos \\
\hline 55 & Kelley Schoo of Business & Estados Unidos \\
\hline 56 & Kellogg Schoo of Management & Estados Unidos \\
\hline 57 & Krannert Graduate School of Management & Estados Unidos \\
\hline 58 & Lancaster University Management School & Reino Unido \\
\hline 59 & Leeds University Business Schoo & Reino Unido \\
\hline 60 & Leonard N Stern School of Business & Estados Unidos \\
\hline 61 & London Business School & Reino Unido \\
\hline 62 & Macouarie Graduate Schoo of Management & Australia \\
\hline 63 & Manchester Business School & Reino Unido \\
\hline 64 & Mannheim Business School & Alemania \\
\hline 65 & Mays Business Schoo & Estados Unidos \\
\hline 66 & Mctombs Schoo of Business & Estados Unidos \\
\hline 67 & Melbourne Business School & Australia \\
\hline 68 & Mendoza College of Business & Estados Unidos \\
\hline 69 & MIT Sloan School of Management & Estados Unidos \\
\hline 70 & Monash Graduate School of Business & Australia \\
\hline 71 & Newcastle University Business School & Reino Unido \\
\hline 72 & Norwegian School of Management B & Noruega \\
\hline 73 & Nottingham University Business School & Reino Unido \\
\hline 74 & Open University Business School & Reino Unido \\
\hline 75 & Owen Graduate School of Management & Estados Unidos \\
\hline 76 & Oxford Said Business School & Reino Unido \\
\hline 77 & Queen's School of Management \& Business & Reino Unido \\
\hline 78 & Robert Emmett McDonough School of Business & Estados Unidos \\
\hline 79 & Robert H. Smith Schoo of Business & Estados Unidos \\
\hline 80 & Rotterdam School of Management & Palses Bajos \\
\hline 81 & Royal Holloway School of Management & Reino Unido \\
\hline 82 & Sauder School of Business & Canadá \\
\hline 83 & Schulich Business School & Canada \\
\hline 84 & SDA Bocconi & Italia \\
\hline 85 & Sheffield University Management School & Reino Unido \\
\hline 86 & Smeal College of Business & Estados Unidos \\
\hline 87 & Solvay Business School & Bélgica \\
\hline
\end{tabular}




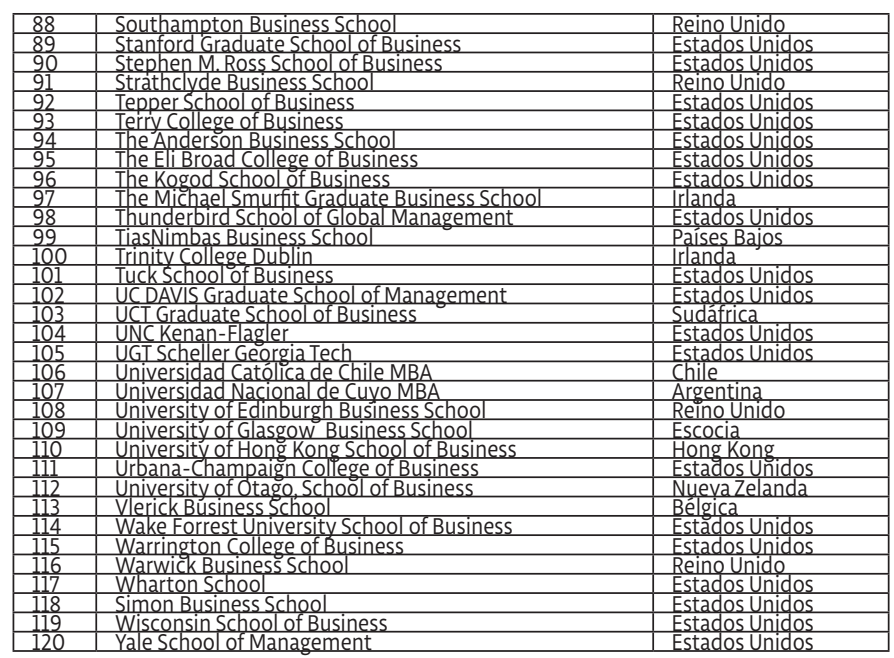

\section{Referencias bibliográficas}

Aranguren, J. L. (1996): Ética y Política. Madrid: Biblioteca Nueva.

Barquero, J.D. y Osterman, P. (2013): Nuevas Fronteras del Management. Barcelona: Furtwanguen.

Boriaud, J. (2016): Nicolás Machiavelo. Buenos Aires: El Ateneo.

Brians, P et al, eds. (1993). Reading About the World, Vol. 1. Washington State University

Ciappei, C. (2006): "Il realismo strategico nel governo d'impresa. Materiali per una pragmatica del valore”. Firenze: Firenze University Press.

Cláusewitz, C. V. (1976): “On War". Princeton: Princeton University Press,

Cleary, T. (1993): “Thunder in the Sky: on the aqcuisition and exersise of power”.Boston: Shambala,

Cleary, T. (2006): "El Alma del Samurai". Barcelona: Kairos.

Dickey, J.S, citado por Stephen R. (2005). Covey, L'ottava regola. Dall'efficacia all'eccellenza ,Milano: Franco Angeli.

Eftimova Bellinger, V. (2017): "Marie von Clausewitz: The Woman behind the Making of On War". Washington: W\&C.

Garrido, F. J. (2010): "El Alma del Estratega". Madrid: McGraw Hill.

Garrido, F. J. (2018): "Modeling the Future”. Oxford: Oxford Strategy Business Press.

Garrido, F. J. (2019): “Guía Elegir un MBA”. Barcelona: Gestión 2000.

Goya y Balbuena (1982): “Bellum Gallicum, con notas de Napoleón Bonaparte”. Carcelona: Iberia.

Griffith, Samuel (1963): “The Art of War”. Oxford: Oxford University Press,

Holden, P. (2000) Ética para managers, Barcelona: Paidós.

Kakabadse, A and M.Morsing. M (Editors) (2006), Corporate Social Responsibility. Reconciling Aspiration with Application, Palgrave: Macmillan. 
Kaushik, R. (2012): "Hinduism and the Ethics of Warfare in South Asia: from antiquity to present”. New York: Cambridge University Press.

Laporte y Marignani (2012): "Ética y Empresa". En Garrido, FJ: "Lo que se Aprende en los Mejores MBA del Mundo”. Barcelona: Gestión 2000..

Lewis, Kay, Kelso \& Larson. (2010). "Was the 2008 Financial Crisis Caused by a Lack of Corporate Ethics?”. Global Journal of Business Research, Vol. 4, No. 2, pp. 77-84, 2010

Melé, D. (2009): "Business Ethics in Action: seeking human excellence in Organizations". London: Palgrave Mcmillan.

Mintzberg, H. (2004) Managers not MBAsManagers not MBAs, ”. New York: Berrett Koehler Momigliano, A. (1954). "Some Observations on Causes of War in Ancient Historiography", Acta Congressu Msadvigiani Proceedings of the Second International Clongress of Classical Sltudies,Copenhagen, 1958,pp. 199-21

Oshima, H. (2011): "La Vía del Samurai”. Madrid: La Sfera de los Libros.

Ricart, J. E. \& Garrido, F. J.. 2015. “Estrategia y Dirección Estratégica: en busca del eslabón perdido". Asian Journal of Business and Management (ISSN: 2321 - 2802) Volume 03 Issue 05, October.

Shamasastry, R. (1915): "Kautilya. Arthashastra”. Bangalore: Government Press, 515-520.

Schimcke, D. (2010): "El Ejecutivo Samurai". Madrid: Edaf.

Sese, M y Rubio, C. (2013): "Yukio Mishima: la ética del samurái en el Japón moderno". Madrid: Alianza Literaria.

Tao Te K. (1993), versión de John C. H. Wood. Madrid: Edaf.

Wood, Neal (2001): “The Art of War”., Cambridge:Da Capo Press. 